\title{
MATRIX-CONTROLLED HYDRAULIC PROPERTIES OF MISSISSIPPIAN AND PENNSYLVANIAN SANDSTONES FROM THE MICHIGAN BASIN
}

U.S. GEOLOGICAL SURVEY

Open-File Report 90-104

MICHIGAN BASIN REGIONAL AQUIFER SYSTEMS ANALYSIS 
MATRIX-CONTROLLED HYDRAULIC PROPERTIES OF MISSISSIPPIAN AND

PBNNSYLVANIAN SANDSTONES FROM THE MICHIGAN BASIN

By D.B. Westjohn, H.W. 01sen, and A.T. Willden

U.S. GEOLOGICAL SURVEY

Open-File Report 90-104

Lansing. Michigan

1990 
DEPARTMENT OF THB INTBRIOR

MANUBL LUJAN. JR. . Secretary

U.S . GEOLOGICAL SURVEY

Da11as L. Peck. Director

For additional information write to:

District Chief U.S. Geological Survey 6520 Mercantile Way. Suite 5 Lansing. Michigan 48911
Copies of this report can be purchased from:

U.S. Geological Survey Books and Open-File Reports Section Federa1 Center. Bldg. 810

Denver. Colorado 80225 


\section{CONTBNTS}

Page

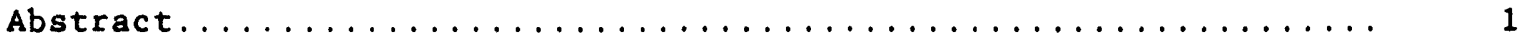

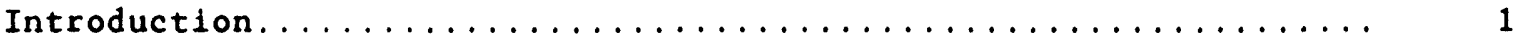

Sampling strategy and sample preparation................ 3

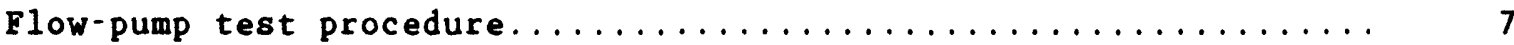

Matrix-controlled hydraulic properties.................. 11

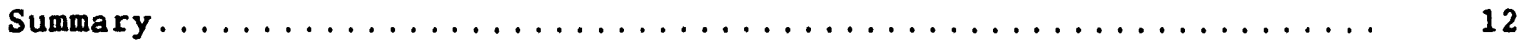

References cited............................... 13

Appendix Plots of hydraulic conductivity as a function of effective stress for Mississippian and Pennsylvanian sandstones from the Michigan basin...............

\section{ILLUSTRATIONS}

Figure 1. Map showing the Lower Peninsula of Michigan. Michigan basin Regional Aquifer System Analysis study area, and the location of drill-holes...................

2. Schematic diagram of flow pump and triaxial apparatus used to measure hydraulic properties..............

\section{TABLBS}

Table 1. Location of diamond-cored drill holes............ 4

2. Sample descriptions..................... 5

3. Summary of porosity and hydraulic-conductivity values for Mississippian and Pennsylvanian sandstone cores from the Michigan basin.................... 


\section{CONVERSION FACTORS AND ABBRBVIATIONS}

For those readers who prefer inch-pound units rather than the metric (International System) units used in this report. the following conversion factors may be used:

Multioly Metric Unit

meter (m)

kilometer ( $\mathrm{km}$ )

square kilometer $\left(\mathrm{km}^{2}\right)$

kilopasca1 (kPa)
By

3.281

0.6214

0.3861

0.145
To Obtain Inch-Pound Unit

foot ( $f t)$

mile (mi)

square mile $\left(\mathrm{mi}^{2}\right)$

pound per square inch

Sea leve1: In this report "sea leve1" refers to the National Geodetic Vertical Datum of 1929 (NGVD of 1929)--a geodetic datum derived from a general adjustment of the first-order level nets of both the United States and Canada. formerly called "Sea Level Datum of 1929." 


\title{
MATRIX-CONTROLLED HYDRAULIC PROPERTIES OF MISSISSIPPIAN AND PENNSYLVANIAN SANDSTONBS FROM THB MICHIGAN BASIN
}

\author{
By D.B. Westjohn, H.W. 01sen, and A.T. Wil1den
}

\begin{abstract}
Hydraulic-conductivity measurements were made of 49 sandstone core plugs using a flow pump and a conventional triaxial confining apparatus. The sandstones tested are samples from the Marshall Sandstone and Grand River and Saginaw Formations, which are the principal bedrock aquifers in the Michigan basin. Sandstones ranging from poorly cemented to well cemented were selected to investigate matrix-controlled hydraulic properties as a function of degree of cementation. Hydraulic conductivities were measured for each sample over a range of effective stress (69 to $827 \mathrm{kilopascals):} \mathrm{hydraulic} \mathrm{conductivities} \mathrm{for} \mathrm{the} \mathrm{sample} \mathrm{suite} \mathrm{range} \mathrm{from}$ $1.9 \times 10^{-2}$ to $2.7 \times 10^{-9}$ centimeters per second. This range of approximately seven orders in magnitude is indicative of local and regional differences in matrix-controlled hydraulic conductivities for Mississippian and Pennsylvanian bedrock aquifers in the Michigan basin.
\end{abstract}

\section{INTRODUCTION}

This report provides preliminary results of an investigation that is designed to determine $K$ (hydraulic conductivity ${ }^{1}$ ) values for selected Michigan basin sandstones as a function of degree of cementation. The sandstone suite tested consists of samples of Mississippian Marshal1 Sandstone from a productive municipal well field (Verona well field. Battle Creek. Michigan), brine-bearing Marshall Sandstone from deep boreholes ( 430 to $480 \mathrm{~m}$ (meters) below land surface) in the central part of the basin, and Pennsylvanian sandstones from the Grand River and Saginaw Formations from five areas of the Lower Peninsula of Michigan where these sandstone aquifers contain freshwater or saline water (fig. 1). These sandstone units are the principal bedrock aquifers in the Michigan basin, and they are one subject of a current hydrogeological investigation being conducted by the U.S. Geological Survey (Michigan basin Regional Aquifer System Analysis).

Measurements of hydraulic conductivity were made using a flow-pump apparatus and methods described by 01 sen and others (1986). The measurements were made at increments of effective stress $(69,138,276$. 552 , and $827 \mathrm{kPa}$ (kilopascals)), and changes in $\mathrm{K}$ as a function of loading

1/ The term hydraulic conductivity indicates vertical hydraulic conductivity. unless otherwise indicated in the text or tables. 


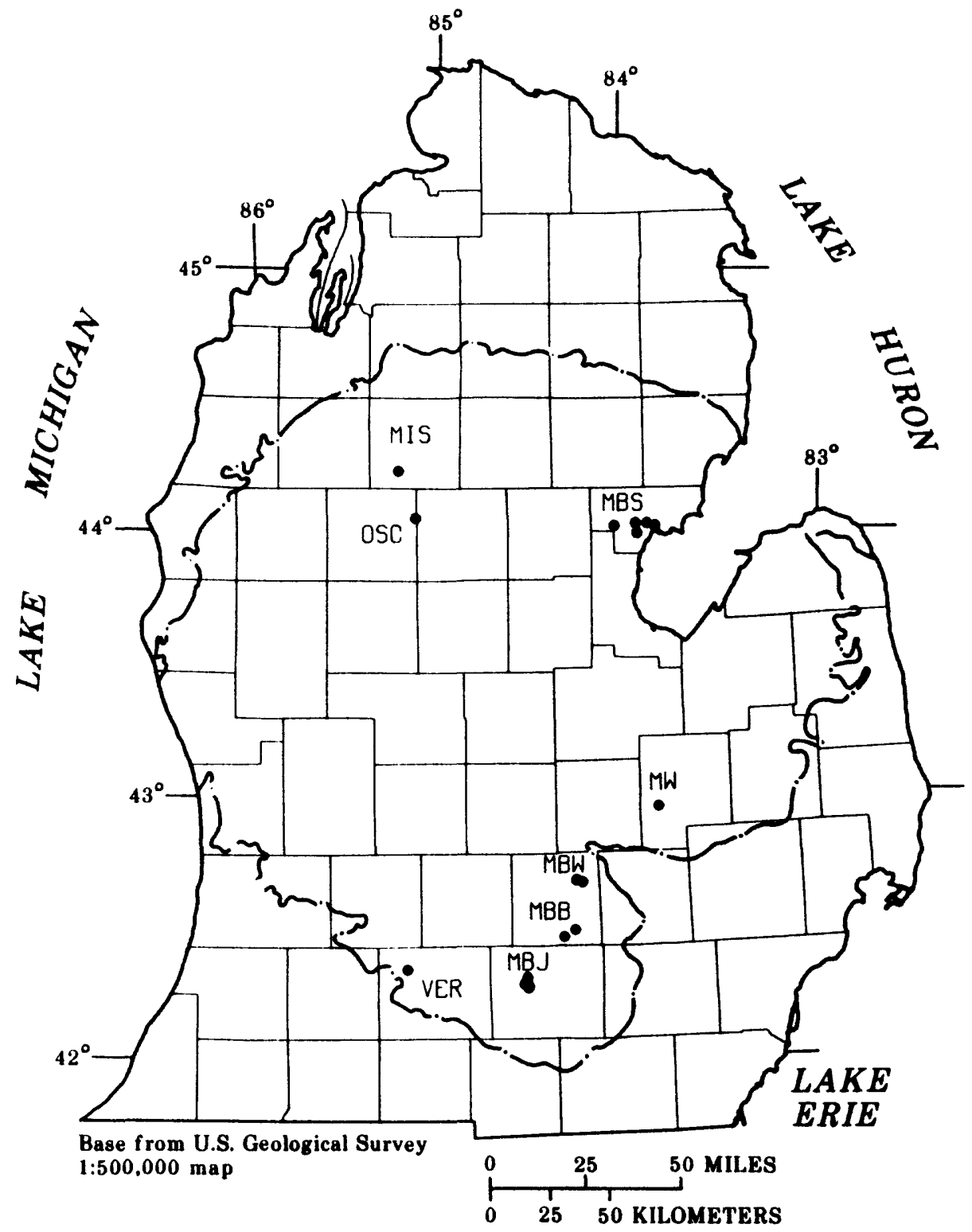

EXPLANATION

- - STUDY AREA-- Boundary of
Marshall Sandstone
VER DRILL HOLE (see table 1)

Figure 1.--The Lower Peninsula of Michigan, Michigan basin Regional Aquifer System Analysis study area, and the location of drill holes. 
and unloading were recorded for most of the sandstones tested. The results of all tests are summarized in the appendix.

The mineralogy and chemistry of the sandstones and cements are currently being investigated using petrographic examinations, $X$-ray fluorescence, $X$-ray diffraction, scanning electron microscopy, and microprobe and isotopic analyses. This work is planned to provide data that will be useful in relating physical properties to the hydraulic properties of the sandstones. The purpose of this report is to publish the hydraulic-conductivity and porosity data.

\section{SAMPLING STRATBGY AND SAMPLE PRBPARATION}

Sandstones from principal bedrock aquifers in the Michigan basin comprise the suite of samples selected for investigation of matrixcontrolled hydraulic properties. The samples tested were selected from existing core collections (Michigan State University and University of Michigan core laboratories: U.S. Geological Survey core collection). A list of locations for the diamond-cored drill holes is provided in table 1.

The sample suite consists of poorly cemented to well-cemented sandstones. Silica, calcite, ankerite, dolomite, kaolinite, and iron oxide are common cements. Chlorite, glauconite, barite, mixed-clays, and gypsum cements are present in some sandstones, but are minor constituents. A general description of the grain size, grain shape, degree of sorting. degree of cementation, and presence or absence of clay for each sample is shown in table 2 .

All samples appear to be unfractured, although the possibility that microfractures may be present in some core plugs cannot be dismissed. The objective of the study is to determine matrix-controlled hydraulic properties, and generate data to provide numerical constraints for groundwater-flow models. The data also are useful for assessing the relative importance of the sandstone matrix, compared to fractures and other secondary openings, in influencing and controlling ground-water flow in sandstone aquifers in the Michigan basin.

The samples tested are from confined and unconfined sandstone aquifers, and were selected from units that contain water that has a wide range of dissolved-solids concentrations (200 to greater than 300,000 milligrams per liter). The samples are from different depths in individual drill holes: the range in depth for the sample suite is 9.0 to $485.2 \mathrm{~m}$.

The sandstone specimens are right cylinders $(2.54 \mathrm{~cm}$ (centimeters) in length and $6.08 \mathrm{~cm}$ in diameter) that were cut from diamond-core plugs. The ends of the core plugs were polished on a $600-\mathrm{grit}$ diamond wheel to reduce irregularities on the cut surfaces. This method of sample preparation allows for accurate volume and porosity determinations. 
Table 1.--Lecation of diamond-cored drill holes

Drill hole identifier: Number of drill hole: prefix abbreviations indicate location and formation. Pennsylvanian sandstones denoted by MW. MBB, MBJ. MBS, and MBW prefixes. Mississippian sandstones denoted by VER, MIS, and OSC prefixes. MW. Swartz Creek area. Genesee. County: MBB. Bunkerhil1 area, Ingham, County: MBJ. Parma area, Jackson, County: MBS. Standish area. Arenac. County: VER, Verona well field. Calhoun, County: MIS. McBain area. Missaukee. County: OSC. Marion area. Osceola, County.

Location: Location of drill hole in township and range subdivision system.

\begin{tabular}{|c|c|c|c|c|c|}
\hline \multirow{2}{*}{$\begin{array}{c}\begin{array}{c}\text { Drill hole } \\
\text { identifier }\end{array} \\
\text { MW- } 7\end{array}$} & \multicolumn{5}{|c|}{ Location } \\
\hline & & Sec & 23 & $\mathrm{~T} 6 \mathrm{~N}$ & R5E \\
\hline MW- 10 & & $\mathrm{Sec}$ & 23 & $\mathrm{~T} 6 \mathrm{~N}$ & R5E \\
\hline$M W-11$ & & Sec & 23 & $\mathrm{~T} 6 \mathrm{~N}$ & R5E \\
\hline MBB - 3 & SW NW & Sec & 21 & T1N & R1E \\
\hline MBB - 5 & SW SW & Sec & 12 & TIN & R1E \\
\hline$M B J-1$ & SW SW & Sec & 23 & T2S & $\mathrm{R} 1 \mathrm{~W}$ \\
\hline MBJ -5 & SW NW & Sec & 22 & T2S & $\mathrm{R} 2 \mathrm{~W}$ \\
\hline MBJ - 10 & SW NW & Sec & 26 & T2S & $\mathrm{R} 2 \mathrm{~W}$ \\
\hline MBJ - 14 & SE SE & Sec & 10 & T2S & $\mathrm{R} 2 \mathrm{~W}$ \\
\hline MBS - 3 & SW SW & Sec & 6 & $\mathrm{~T} 18 \mathrm{~N}$ & R5E \\
\hline MBS - 13 & SE SE & $\mathrm{Sec}$ & 24 & T19N & R4B \\
\hline MBS - 15 & SW NW & Sec & 30 & T19N & R4E \\
\hline MBS - 18 & SE & Sec & 21 & T19N & R4E \\
\hline MBS - 20 & SW NW & Sec & 25 & T19N & R5E \\
\hline MBW- 4 & NW NE & Sec & 8 & T3N & R2B \\
\hline MBW- 5 & SE SE & Sec & 1 & T3N & RIE \\
\hline VER - 4D & SE SE & Sec & 32 & T1S & $R 7 W$ \\
\hline VER-6D & NE NE & Sec & 5 & T2S & R7W \\
\hline MIS $^{1}$ & NE NW & Sec & 8 & $\mathrm{~T} 21 \mathrm{~N}$ & $R 7 W$ \\
\hline $\mathrm{osc}^{2}$ & SE NE & Sec & 12 & T19N & $R 7 W$ \\
\hline
\end{tabular}

$1 /$ Michigan Department of Natural Resources oil and gas permit number 25594 .

2/ Michigan Department of Natural Resources oil and gas permit number 26002 . 


\section{Table 2. - Sample descriptions}

Drill hole identifier: Number of drill hole. prefix abbreviations indicate location. MW. Swartz Creek area. Genesee. County: MBB. Bunkerh111 area, Ingham, County: MBJ. Parma area, Jackson. County: MBS, Standish area. Arenac. County: VER, Verona well fleld. Calhoun. County: MIS. McBain area. Missaukee. County: OSC, Marion area, Osceola. County.

Grain size: Size range of grains in sandstone matrix. Bstimated using binocular microscope or hand lens by comparing sample grain sizes to U.S. Geological Survey sand grain size indicator. Abbreviations based on classification scheme of Blatt and others, 1972. p. 46.: v. very: $f$, fine: m, medium: c, coarse.

Degree of sorting: Degree of sorting of sand grains. based on comparison with sorting comparators (Swanson, 1981, p. III-10, and p.III-11).

Abbreviations: $v$, very: $p$, poor: m, moderate: $w$, well.

Clay cement: Denotes presence $(y)$ or absence $(n)$ of clay cement.

Clay in pores: Denotes the presence ( $y$ ) or absence $(n)$ of clay that fills some or all pores.

Angularity: Range of shape, based on degree of roundness comparators (Swanson, 1981, p. III-14). Abbreviations: a, angular; sa, subangular: sr, subrounded: $r$, rounded.

Degree of cementation: Qualitative observation. Abbreviations: v, very: p. poor: m, moderate: $w$, well.

\begin{tabular}{|c|c|c|c|c|c|c|c|}
\hline $\begin{array}{c}\text { Drill } \\
\text { hole } \\
\text { identifier }\end{array}$ & $\begin{array}{l}\text { Depth. } \\
\text { in } \\
\text { meters }\end{array}$ & $\begin{array}{c}\text { Grain } \\
\text { size }\end{array}$ & $\begin{array}{c}\text { Degree } \\
\text { of } \\
\text { sorting }\end{array}$ & $\begin{array}{c}\text { Clay } \\
\text { cement }\end{array}$ & $\begin{array}{l}\text { Clay } \\
\text { in } \\
\text { pores }\end{array}$ & Angularity & $\begin{array}{c}\text { Degree } \\
\text { of } \\
\text { cementation }\end{array}$ \\
\hline MW - 7 & 53.1 & $f-v c$ & p & $y$ & y & $r$ & $\mathbf{w}$ \\
\hline MW- 10 & 54.9 & $m-c$ & $\mathbf{m}$ & $\mathbf{n}$ & y & $s r-r$ & $\mathbf{w}$ \\
\hline MW - 11 & 54.9 & $m-c$ & $\mathbf{m}$ & y & y & $s r-r$ & $\mathbf{m}$ \\
\hline MBB - 3 & 36.1 & $f$ & $\mathbf{w}$ & $\mathbf{n}$ & y & $s a-s r$ & mw \\
\hline MBB - 3 & 73.8 & $v f-f$ & $w$ & $\mathbf{n}$ & $y$ & $s a-s r$ & $w$ \\
\hline MBB - 5 & 21.6 & $v f-f$ & $w$ & $\mathbf{n}$ & $\mathbf{n}$ & sr & vw \\
\hline MBB - 5 & 27.8 & $f-m$ & $\mathbf{m}$ & $y$ & y & $s a-s r$ & $\mathbf{w}$ \\
\hline MBJ - 1 & 30.7 & $v f \cdot f$ & $\mathbf{m}$ & $y$ & y & $a-s r$ & $\mathbf{w}$ \\
\hline MBJ - 5 & 14.2 & m & $\mathbf{m}$ & $\mathrm{n}$ & y & $s r-r$ & $\mathbf{m}$ \\
\hline MBJ - 10 & 9.6 & $v f-f$ & $\mathbf{m}$ & $\mathbf{n}$ & y & sa-sr & $\mathbf{m}$ \\
\hline MBJ - 10 & 17.0 & $m-c$ & $\mathbf{w}$ & $y$ & $\mathrm{n}$ & $s a-r$ & $\mathrm{p}$ \\
\hline MBJ - 10 & 25.0 & $\mathbf{m}$ & $w$ & $y$ & y & $\mathbf{r}$ & $\mathrm{p}$ \\
\hline MBJ - 10 & 28.4 & $v f-m$ & $p$ & $y$ & y & $a-s r$ & mw \\
\hline MBJ - 10 & 39.2 & $\mathbf{f}$ & $w$ & n & n & sr & vw \\
\hline
\end{tabular}


Tab1e 2.--Sample descriptions--Continued

\begin{tabular}{|c|c|c|c|c|c|c|c|}
\hline $\begin{array}{c}\text { Drill } \\
\text { hole } \\
\text { identifier }\end{array}$ & $\begin{array}{l}\text { Depth, } \\
\text { in } \\
\text { meters }\end{array}$ & $\begin{array}{r}\text { Grain } \\
\text { size }\end{array}$ & $\begin{array}{c}\text { Degree } \\
\text { of } \\
\text { sorting }\end{array}$ & $\begin{array}{c}\text { Clay } \\
\text { cement }\end{array}$ & $\begin{array}{l}\text { Clay } \\
\text { in } \\
\text { pores }\end{array}$ & Angularity & $\begin{array}{c}\text { Degree } \\
\text { of } \\
\text { cementation }\end{array}$ \\
\hline MBJ - 10 & 48.8 & $f-v f$ & $\mathbf{m}$ & $y$ & $\mathbf{y}$ & $\mathbf{s r}-\mathbf{r}$ & m \\
\hline $\mathrm{MBJ}-14$ & 30.6 & $v f-f$ & $\mathbf{m}$ & $\mathbf{y}$ & $\mathbf{y}$ & $a-s r$ & $\mathbf{w}$ \\
\hline $\mathrm{MBJ}-14$ & 61.4 & $f$ & $\mathbf{w}$ & $y$ & $y$ & $\mathbf{a}-\mathbf{s a}$ & $\mathbf{w}$ \\
\hline $\mathrm{MBJ}-14$ & 98.7 & $f-v f$ & $\mathbf{m}$ & $\mathbf{y}$ & $\mathbf{y}$ & $\mathbf{s a}-\mathbf{r}$ & m \\
\hline MBS - 3 & 20.1 & $\mathbf{m}$ & $w$ & $\mathrm{n}$ & $\mathrm{n}$ & $s r-r$ & $\mathbf{m}$ \\
\hline MBS - 3 & 25.0 & $f-m$ & $w$ & $\mathrm{n}$ & $\mathbf{n}$ & $a-s r$ & $p$ \\
\hline MBS - 3 & 33.4 & $f-m$ & $w$ & $y$ & $\mathbf{y}$ & $s r-r$ & mw \\
\hline MBS - 3 & 41.4 & $f-m$ & $\mathbf{m}$ & $n$ & $n$ & $\mathbf{s a - s}$ & $\mathbf{m}$ \\
\hline MBS - 13 & 12.3 & $m-c$ & $\mathbf{m}$ & $\mathrm{n}$ & $\mathbf{y}$ & $s r-r$ & mw \\
\hline MBS - 13 & 17.0 & $f-v c$ & vp & $\mathbf{y}$ & $y$ & $a-r$ & vp \\
\hline MBS - 15 & 9.3 & $f-c$ & $p$ & y & $y$ & $8 a-s r$ & m \\
\hline MBS - 15 & 46.9 & $\mathbf{m}-\mathbf{c}$ & mw & $y$ & $y$ & $s r-r$ & w \\
\hline MBS - 15 & 67.0 & $f$ & $w$ & $n$ & y & $s a-s r$ & $\mathbf{m}$ \\
\hline MBS - 18 & 9.0 & $f \cdot m$ & $\mathbf{m}$ & $y$ & $y$ & $s r-r$ & $\mathbf{m}$ \\
\hline MBS - 20 & 9.0 & $f-v c$ & $\mathbf{v p}$ & y & y & $s r-r$ & $p$ \\
\hline MBS - 20 & 15.7 & c & $\mathbf{w}$ & $\mathrm{n}$ & $n$ & $r$ & $p-m p$ \\
\hline MBS - 20 & 17.0 & c & $w$ & $\mathrm{n}$ & $\mathrm{n}$ & $\mathbf{r}$ & $p$ \\
\hline MBW - 4 & 19.1 & $v f-f$ & $\mathbf{m}$ & $\mathbf{y}$ & $y$ & $s a-s r$ & mw \\
\hline MBW - 4 & 34.0 & $f-m$ & $\mathbf{m}$ & y & y & $s a-r$ & $\mathbf{m}$ \\
\hline MBW- 5 & 26.2 & $\mathbf{m}$ & $\mathbf{m}$ & y & $y$ & $\mathbf{a}-\mathbf{s} \mathbf{a}$ & $w$ \\
\hline MBW - 5 & 36.4 & $f-m$ & $\mathbf{m}$ & $n$ & $\mathrm{n}$ & sa & $w$ \\
\hline MBW - 5 & 41.4 & $f-m$ & $\mathrm{p}$ & $\mathrm{n}$ & $\mathbf{y}$ & $\mathbf{s a}$ & vw \\
\hline VER - 4D & 13.3 & $v f-f$ & $\mathbf{m}$ & $\mathrm{n}$ & $n$ & $s a-s r$ & vw \\
\hline VER - 4D & 23.5 & $v f-f$ & $\mathbf{m}$ & $\mathrm{n}$ & $\mathrm{n}$ & $s a-s r$ & vw \\
\hline VER - 4D & 28.7 & $v f-f$ & $\mathbf{m}$ & $\mathrm{n}$ & $\mathrm{n}$ & $s a-s r$ & $\mathbf{v w}$ \\
\hline VER - 4D & 39.5 & $v f-f$ & $\mathbf{m}$ & $\mathrm{n}$ & $\mathrm{n}$ & $s a-s r$ & vw \\
\hline VER-6D & 16.4 & $v f-f$ & $\mathbf{m}$ & $\mathrm{n}$ & $\mathrm{n}$ & $s a-s r$ & vw \\
\hline VER - 6D & 18.2 & $v f-f$ & $\mathbf{m}$ & $\mathrm{n}$ & $\mathrm{n}$ & $s a-s r$ & vw \\
\hline VER - 6D & 22.2 & $v f-f$ & $\mathbf{m}$ & $\mathrm{n}$ & $n$ & $s a-s r$ & vw \\
\hline MIS & 450.3 & $\mathbf{m}$ & $w$ & $\mathbf{y}$ & $y$ & $a-r$ & vw \\
\hline MIS & 452.4 & $f-m$ & $\mathrm{mw}$ & $y$ & $y$ & $s a-s r$ & $\mathrm{mw}$ \\
\hline MIS & 454.3 & $v f-f$ & mw & $n$ & y & sa & $\mathbf{w}$ \\
\hline MIS & 485.2 & $\mathbf{m}$ & $w$ & $n$ & y & $a-r$ & vw \\
\hline OSC & 464.5 & $v f-f$ & mw & $y$ & $y$ & $a-s r$ & vw \\
\hline osc & 473.8 & $\mathbf{v f}-\mathbf{f}$ & $\mathrm{mw}_{\mathrm{w}}$ & $y$ & $y$ & $a-s r$ & vw \\
\hline
\end{tabular}


Porosities (table 3 ) were calculated by comparing the mass of the dry sample to the water-saturated mass of the sample.

\section{FLOW-PUMP TBST PROCEDURB}

Values of hydraulic conductivity were determined over a range of effective stress for each sample. Changes in the sample during loading and unloading were recorded during most tests: different values of hydraulic conductivity are plotted as a function of effective stress (appendix and table 3). The procedure to obtain this data is described by olsen (1966). and the apparatus and applications of the flow-pump method are summarized by 01 sen and others (1986). These publications should be referred to for details of the test procedure.

The technique involves using a flow pump to induce a constant volume of fluid at one end of a sample. The sample ts enclosed in a triaxial confining apparatus (01sen and others, 1986), which allows for the application and maintenance of effective stress. The effective stress is transmitted to the sample through a surgical-rubber membrane that surrounds the sandstone cylinder. A hydraulic head is generated at one end of a test sample. while a constant pore pressure is maintained at the opposite end. An inttial transient record of hydraulic head and the subsequent steadystate condition are recorded as a function of time using a differential pressure transducer connected to a strip-chart recorder. The effective stress is controlled using a second flow pump to increase or decrease fluid pressure in the cell that surrounds the sample. The equipment used to measure hydraulic conductivity is illustrated in a simplified schematic diagram (fig. 2). 
Table 3. - Summary of poresity and hydraulic conductivity values for Yississipotan and Pennsylyanian sandstone cores from the Hichiean basin

Drill hole identifier: Number of drill hole. prefix abbreviatione indicate location. MW. Swartz Creek area, Genesee, County: MBB, Bunkerhill area, Ingham, County: MBJ. Parma area, Jackson, County: MBS, Standish area, Arenac. County: VER. Verona well field. Calhoun. County: MIS. McBain area. Missaukee. County: OSC. Marion area, Osceola. County. See figure 1 for locations.

Hydraulic conductivity, $\mathrm{K}(\mathrm{cm} / \mathrm{s}$ (centimeters per second)): Measured hydraulic conductivity from flow-pump test, in $\mathrm{cm} / \mathrm{s}$; all $\mathrm{K}$ values represent vertical hydraulic conductivity. except drill hole numbers with an asterisk (*). The reported $K$ values for these samples are horizontal hydraulic conductivities.

\begin{tabular}{|c|c|c|c|c|}
\hline $\begin{array}{c}\text { Drill } \\
\text { hole } \\
\text { identifier }\end{array}$ & $\begin{array}{l}\text { Depth. } \\
\text { in } \\
\text { meters }\end{array}$ & $\begin{array}{l}\text { Porosity. } \\
\text { in } \\
\text { percent }\end{array}$ & $\begin{array}{l}\text { Hydraulic } \\
\text { Minimum } \\
\text { value }\end{array}$ & $\begin{array}{l}\text { ctivity, } K(\mathrm{~cm} / \mathrm{s}) \\
\text { Maximum } \\
\text { value }\end{array}$ \\
\hline MW-7 & 53.1 & 22.56 & $1.1 \times 10^{-3}$ & $1.3 \times 10^{-3}$ \\
\hline MW - 10 & 54.9 & 19.84 & $6.8 \times 10^{-5}$ & $6.8 \times 10^{-4}$ \\
\hline MW - 11 & 54.9 & 21.04 & $2.9 \times 10^{-3}$ & $5.9 \times 10^{-3}$ \\
\hline MBB - 3 & 36.1 & 18.50 & $3.1 \times 10^{-4}$ & $3.3 \times 10^{-4}$ \\
\hline MBB - 3 & 73.8 & 19.87 & $2.7 \times 10^{-7}$ & $4.8 \times 10^{-5}$ \\
\hline MBB - 5 & 21.6 & 3.20 & $2.7 \times 10^{-9}$ & $4.2 \times 10^{-8}$ \\
\hline MBB - 5 & 27.8 & 18.22 & $2.0 \times 10^{-6}$ & $4.1 \times 10^{-6}$ \\
\hline $\mathrm{MBJ}-1$ & 30.7 & 17.34 & $9.3 \times 10^{-7}$ & $4.7 \times 10^{-5}$ \\
\hline MBJ - 5 & 14.2 & 24.24 & $1.4 \times 10^{-3}$ & $2.2 \times 10^{-3}$ \\
\hline MBJ - 10 & 9.6 & 19.91 & $2.5 \times 10^{-4}$ & $3.0 \times 10^{-4}$ \\
\hline $\mathrm{MBJ}-10$ & 17.0 & 20.19 & $2.9 \times 10^{-3}$ & $2.2 \times 10^{-3}$ \\
\hline MBJ - 10 & 25.0 & 19.20 & $3.3 \times 10^{-4}$ & $4.1 \times 10^{-4}$ \\
\hline MBJ - 10 & 28.4 & 19.80 & $9.9 \times 10^{-6}$ & $3.2 \times 10^{-5}$ \\
\hline MBJ - 10 & 39.2 & 11.60 & $4.1 \times 10^{-8}$ & $7.8 \times 10^{-7}$ \\
\hline MBJ - 10 & 48.8 & 15.45 & $8.0 \times 10^{-8}$ & $9.1 \times 10^{-7}$ \\
\hline MBJ - 14 & 30.6 & 14.82 & $5.2 \times 10^{-7}$ & $2.8 \times 10^{-6}$ \\
\hline MBJ - 14 & 61.4 & 25.59 & $9.9 \times 10^{-7}$ & $3.3 \times 10^{-6}$ \\
\hline $\mathrm{MBJ}-14$ & 98.7 & 23.07 & $9.0 \times 10^{-6}$ & $1.9 \times 10^{-5}$ \\
\hline MBS - 3 & 20.1 & 24.15 & $2.6 \times 10^{-3}$ & $3.5 \times 10^{-3}$ \\
\hline MBS - 3 & 25.0 & 24.71 & $2.7 \times 10^{-3}$ & $3.1 \times 10^{-3}$ \\
\hline MBS - 3 & 33.4 & 24.92 & $1.2 \times 10^{-3}$ & $1.4 \times 10^{-3}$ \\
\hline MBS - 3 & 41.4 & 33.88 & $6.2 \times 10^{-4}$ & $1.2 \times 10^{-3}$ \\
\hline MBS - 13 & 12.3 & 23.02 & $3.5 \times 10^{-3}$ & $9.5 \times 10^{-3}$ \\
\hline MBS - 13 & 17.0 & 22.08 & $4.0 \times 10^{-3}$ & $6.4 \times 10^{-3}$ \\
\hline MBS - 15 & 9.3 & 22.11 & $4.7 \times 10^{-4}$ & $9.5 \times 10^{-4}$ \\
\hline MBS - 15 & 46.9 & 18.49 & $1.6 \times 10^{-3}$ & $1.8 \times 10^{-3}$ \\
\hline MBS - 15 & 67.0 & 21.58 & $6.3 \times 10^{-4}$ & $7.0 \times 10^{-4}$ \\
\hline MBS - 18 & 9.0 & 22.89 & $1.7 \times 10^{-3}$ & $2.1 \times 10^{-3}$ \\
\hline
\end{tabular}


Table 3. - Summary of perosity and hydraulic conductivity values for Mrsisgippian and Pennsylyanian sandstone cores from the Michisan basin--Continued

\begin{tabular}{|c|c|c|c|c|}
\hline \multirow{3}{*}{$\begin{array}{c}\text { Drill } \\
\text { hole } \\
\text { identifier } \\
\text { MBS-20 }\end{array}$} & \multirow{3}{*}{$\begin{array}{c}\begin{array}{c}\text { Depth, } \\
\text { In } \\
\text { meters }\end{array} \\
9.0\end{array}$} & \multirow{3}{*}{$\begin{array}{c}\begin{array}{c}\text { Porosity. } \\
\text { in } \\
\text { percent }\end{array} \\
18.96\end{array}$} & \multicolumn{2}{|c|}{ Hydraulic conductivity, $\mathrm{K}(\mathrm{cm} / \mathrm{s})$} \\
\hline & & & $\begin{array}{l}\text { Minimum } \\
\text { value }\end{array}$ & $\begin{array}{c}\text { Maximum } \\
\text { value }\end{array}$ \\
\hline & & & $3.2 \times 10^{-3}$ & $5.0 \times 10^{-3}$ \\
\hline MBS - 20 & 15.7 & 24.38 & $3.1 \times 10^{-3}$ & $4.2 \times 10^{-3}$ \\
\hline MBS - 20 & 17.0 & 22.52 & $1.9 \times 10^{-2}$ & $2.5 \times 10^{-2}$ \\
\hline MBW -4 & 19.1 & 14.77 & $3.6 \times 10^{-8}$ & $1.6 \times 10^{-6}$ \\
\hline MBW -4 & 34.0 & 18.48 & $9.2 \times 10^{-6}$ & $1.5 \times 10^{-6}$ \\
\hline MBW- 5 & 26.2 & 19.83 & $8.2 \times 10^{-6}$ & $1.9 \times 10^{-5}$ \\
\hline MBW- 5 & 36.4 & 4.26 & $3.9 \times 10^{-8}$ & $1.2 \times 10^{-6}$ \\
\hline MBW- 5 & 41.4 & 4.87 & $4.1 \times 10^{-9}$ & $1.8 \times 10^{-6}$ \\
\hline VER - 4D & 13.3 & 22.79 & $2.8 \times 10^{-6}$ & $4.9 \times 10^{-6}$ \\
\hline VER - 4D & 23.5 & 21.89 & $5.6 \times 10^{-6}$ & $9.4 \times 10^{-6}$ \\
\hline VER - 4D & 28.7 & 21.96 & $4.7 \times 10^{-6}$ & $9.7 \times 10^{-6}$ \\
\hline VER - 4D & 39.5 & 24.87 & $2.8 \times 10^{-6}$ & $8.2 \times 10^{-6}$ \\
\hline VER - 6D & 16.4 & 18.48 & $3.4 \times 10^{-6}$ & $4.1 \times 10^{-6}$ \\
\hline VER - 6D & 18.2 & 23.17 & $5.4 \times 10^{-5}$ & $6.6 \times 10^{-5}$ \\
\hline VBR - 6D & 22.2 & 21.16 & $3.6 \times 10^{-6}$ & $6.7 \times 10^{-6}$ \\
\hline MIS * & 450.3 & 21.99 & $3.8 \times 10^{-4}$ & $5.7 \times 10^{-4}$ \\
\hline MIS * & 452.4 & 18.82 & $2.5 \times 10^{-4}$ & $2.8 \times 10^{-4}$ \\
\hline MIS * & 454.3 & 20.77 & $1.7 \times 10^{-4}$ & $1.8 \times 10^{-4}$ \\
\hline MIS* & 485.2 & 17.71 & $1.4 \times 10^{-4}$ & $1.7 \times 10^{-4}$ \\
\hline OSC & 464.5 & 16.69 & $6.1 \times 10^{-6}$ & $9.8 \times 10^{-6}$ \\
\hline OSC & 473.8 & 16.44 & $1.6 \times 10^{-5}$ & $1.8 \times 10^{-5}$ \\
\hline
\end{tabular}




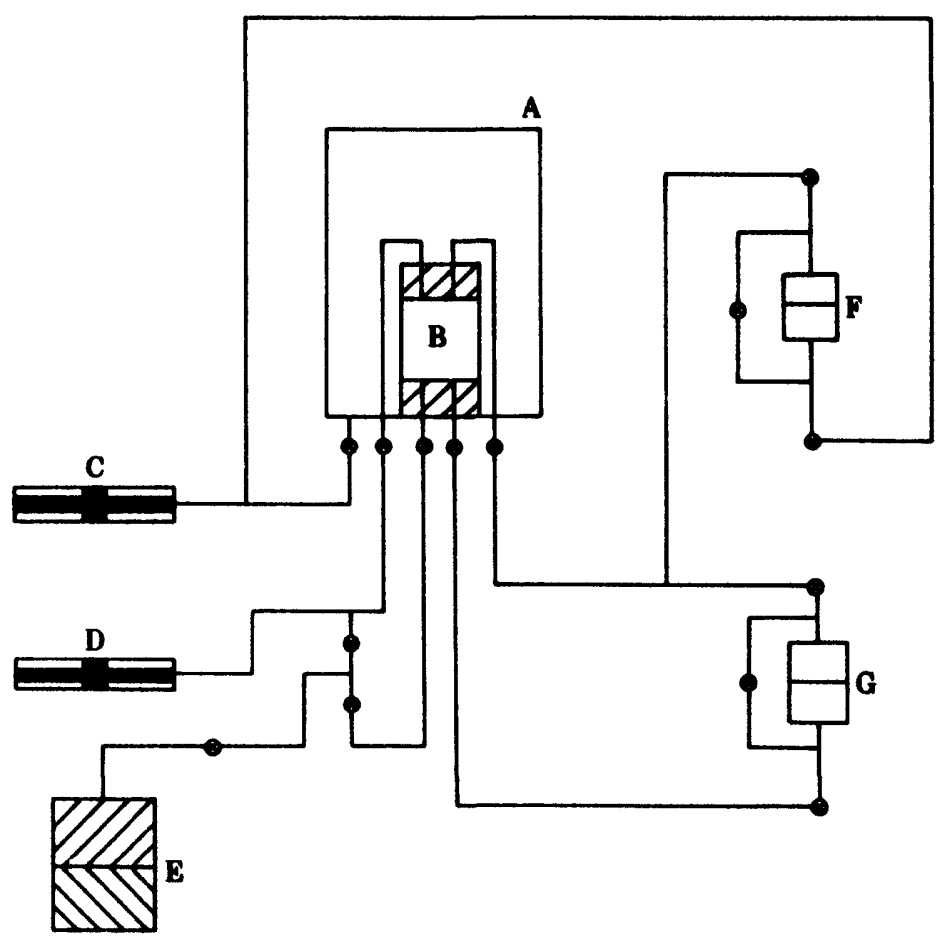

EXPLANATION
A conventional triax
B TEST 8AMPLE
C FLOW-PUMP ACTUATOR
D FLOW-PUMP ACTUATOR
E BeLLOPRAM PREgSURE REgULATOR
F differential PREgBURE TRANBdUCER
G DIPFERENTIAL PREgBURE TRAN8DUCER
- VALVE

Figure 2.--Schematic diagram of flow pump and triaxial apparatus used to measure hydraulic properties. (See 0lsen and others, 1986. for details of equipment layout and test procedures.)

The hydraulic conductivity at a particular effective stress is calculated using Darcy's Law:

$$
K=\frac{L(0) \gamma_{w}}{A(\Delta P)}
$$

where

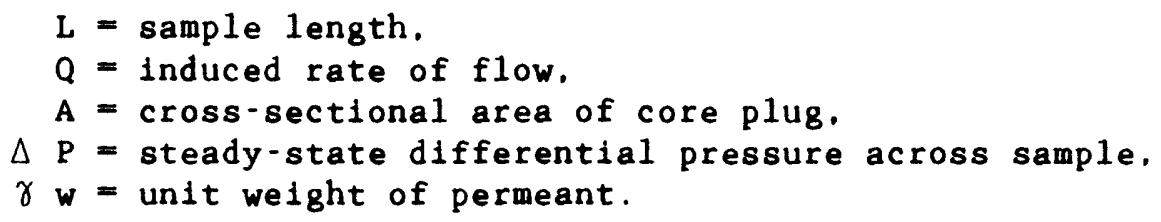




\section{MATRIX-CONTROLLED HYDRAULIC PROPBRT IBS}

Inspection of table 3 and the plots in the appendix show that there is a substantial range in matrix-controlled hydraulic conductivities of Michigan basin sandstones. The range $\left(1.9 \times 10^{-2}\right.$ to $2.7 \times 10^{-9} \mathrm{~cm} / \mathrm{s}$ exceeds the range of hydraulic conductivities listed by Freeze and Cherry (1979. p. 29) for sandstones, and the lower $K$ values overlap with the hydraulic conductivities listed for shale and unfractured crystalline rocks (Freeze and Cherry, 1979, p. 29).

Most samples exhibit a slight reduction in hydraulic conductivity with an increase in effective stress, but there appears to be a threshold at approximately $276 \mathrm{kPa}$ where additional effective stress has little effect. This change is attributed to the closure of microfractures at low effective stress rather than being related to compressibility. There are instances where hydraulic conductivity decreases over the range of effective stress ( 69 to $827 \mathrm{kPa}$ ), and, in one case, the decrease exceeds two orders in magnitude (see appendix, sample MBW-5. $41.4 \mathrm{~m}$ ). This reduction in hydraulic conductivity is probably the result of sample compressibility as indicated by the rebound observed during unloading. For samples that exhibit this characteristic, the unloading curve closely matches the loading curve. These samples are cemented with kaolinite, and pore space also contains kaolinite and other clay minerals.

The range of hydraulic conductivity values for Pennsylvanian sandstones $\left(1.9 \times 10^{-2}\right.$ to $\left.2.7 \times 10^{-9} \mathrm{~cm} / \mathrm{s}\right)$ substantially exceeds the range in hydraulic conductivity of Mississippian sandstones $\left(3.8 \times 10^{-4}\right.$ to $2.8 \mathrm{X}$ $10^{-6} \mathrm{~cm} / \mathrm{s}$ ). In general. these differences are a function of cement type: silica and carbonate cements are common in Mississippian sandstones. whereas cement in Pennsylvanian sandstones is diverse in terms of mineralogy.

Matrix-controlled hydraulic conductivity values for the Marshall Sandstone at the Verona well field have a narrow range $\left(5.4 \times 10^{-5}\right.$ to $3.4 \mathrm{X}$ $\left.10^{-6} \mathrm{~cm} / \mathrm{s}\right)$, and the average $\left(3.9 \times 10^{-6} \mathrm{~cm} / \mathrm{s}\right)$ is nearly four orders in magnitude less than the minimum hydraulic conductivity $\left(5.3 \times 10^{-2} \mathrm{~cm} / \mathrm{s}\right)$ reported by Grannemann and Twenter (1985). The range in horizontal hydraulic conductivity $\left(1.9 \times 10^{-1} \mathrm{~cm} / \mathrm{s}\right.$ for the lower sandstone, to $5.3 \mathrm{X}$ $10^{-2} \mathrm{~cm} / \mathrm{s}$ for the upper sandstone) determined by aquifer tests at the Verona well field (Grannemann and Twenter. 1985) includes matrix- and fractured-controlled components. It is suggested that hydraulic conductivity measured by aquifer tests at the well field (Grannemann and Twenter. 1985) reflects the contribution of fractures and bedding-plane partings, and that the sandstone matrix has a negligible effect. 


\section{SUMMARY}

The Marsha11 Sandstone and Grand River and Saginaw Formations, which are the principal bedrock aquifers in the Michigan basin. exhibit a wide range in matrix-controlled hydraulic properties. The differences observed are predominantly a function of cement type and degree of cementation. The presence of clay cement and clay in pore spaces controls the compressibility of some sandstones tested, and a decrease in hydraulic conductivity corresponds to an increase in confining stress where kaolinite and other clay minerals are the principal cements. The sandstones examined are heterogeneous, and the range in hydraulic conductivity values exceeds the range commonly 1isted for sandstones (Freeze and Cherry, 1979, p. 29). The matrix-controlled hydraulic conductivities provided with this report are suggested to be characteristic of local and regional differences in hydraulic properties for Mississippian and Pennsylvanian sandstones in the Michigan basin. 


\section{REFBRENCES CITBD}

Blatt, Harvey, Middleton. Gerard, and Murray, Raymond, 1972, Origin of Sedimentary Rocks: Prentice-Hall, Inc.. Bnglewood Hills, New Jersey. $634 \mathrm{p}$.

Freeze, R.A., and Cherry, J.A., 1979, Groundwater: Prentice-Hall, Inc.. New Jersey. $604 \mathrm{p}$.

Grannemann, N.G., and Twenter, F.R., 1985, Geohydrology and ground-water flow at Verona well field. Battle Creek. Michigan: U.S. Geological Survey Water-Resources Investigations Report 85-4056. 54 p.

01sen, H.W.. 1966. Darcy's Law in saturated kaolinite: Water Resources Research, v. 2, no. 6, p. 287-295.

01sen, H.W. Morin, R.H., and Nichols, R.W., 1986. Flow pump applications in triaxial testing, in Donaghe. R.T.. and others, eds.. Advanced Triaxial Testing of Soll and Rock: American Society for Testing and Materials. Philadelphia. p. 68-81.

Swanson. R.G., 1981. Sample Examination Manua1: The American Association of Petroleum Geologists. Tulsa, Oklahoma. $35 \mathrm{p}$. 


\section{APPENDIX}

Plots of hydraulic conductivity as a function of effective stress for Mississippian and Pennsylvanian sandstones from the Michigan basin. 

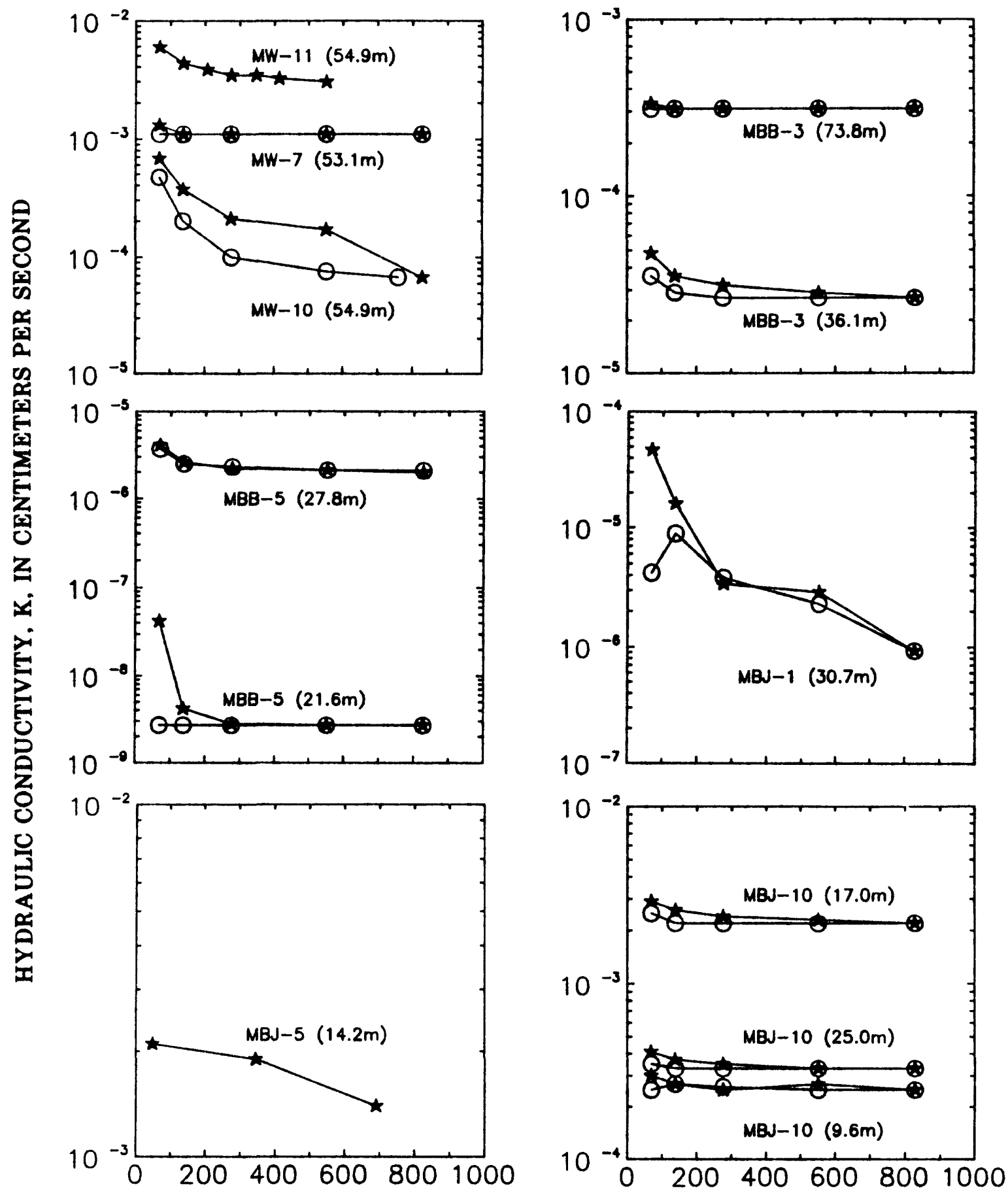

EFFECTIVE STRESS, IN KILOPASCALS

\section{EXPLANATION \\ - LOADING \\ O UNLOADING}

MBJ-10 (9.6m) DRILI. HOLE IDENTIFIER --

Depth, in meters 

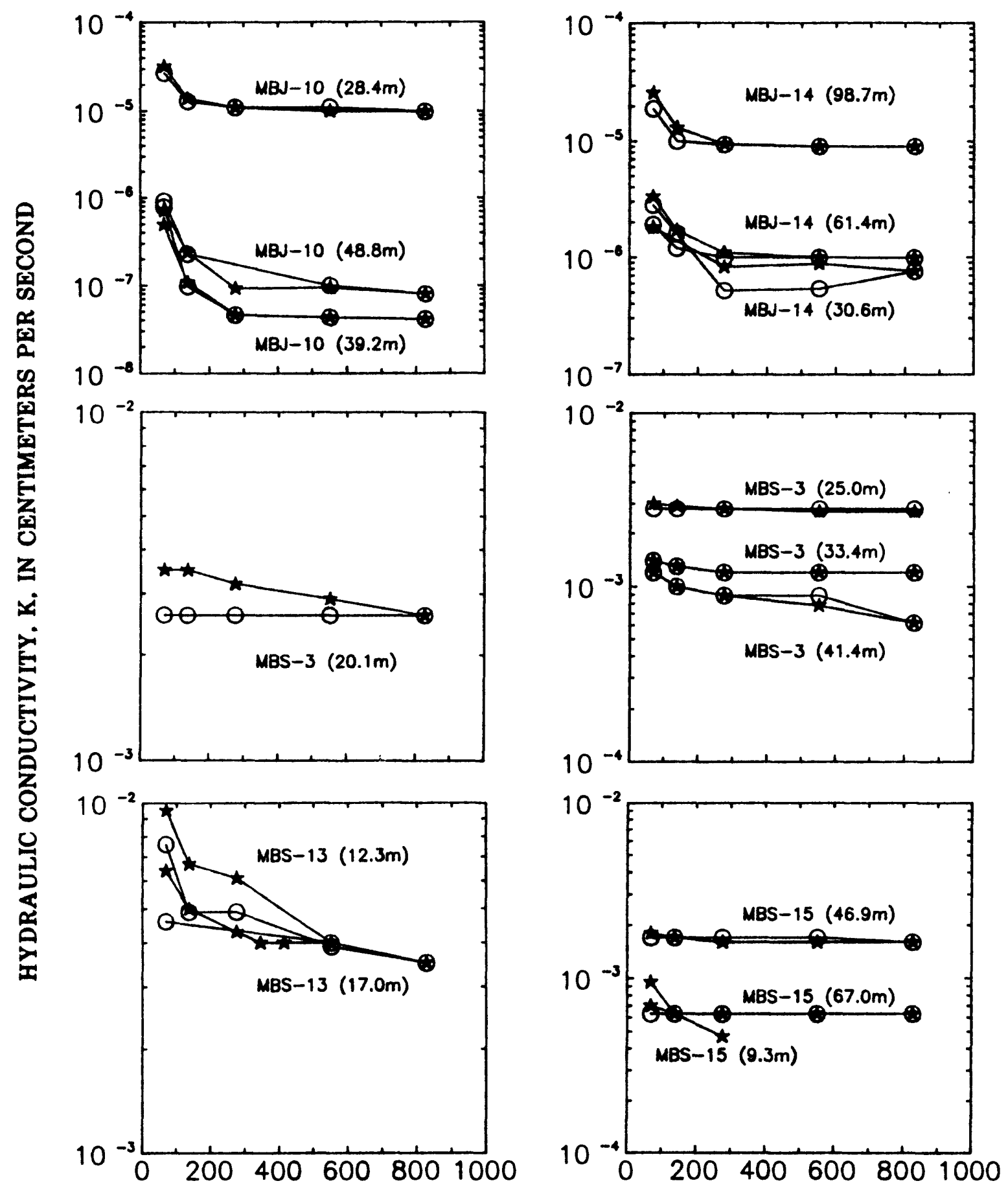

EFFECTIVE STRESS, IN KILOPASCALS

\section{EXPLANATION \\ - LOADING \\ O UNLOADING}

MBS- 15 (9.3m) DRILL HOLE IDENTIFIER --

Depth, in meters 

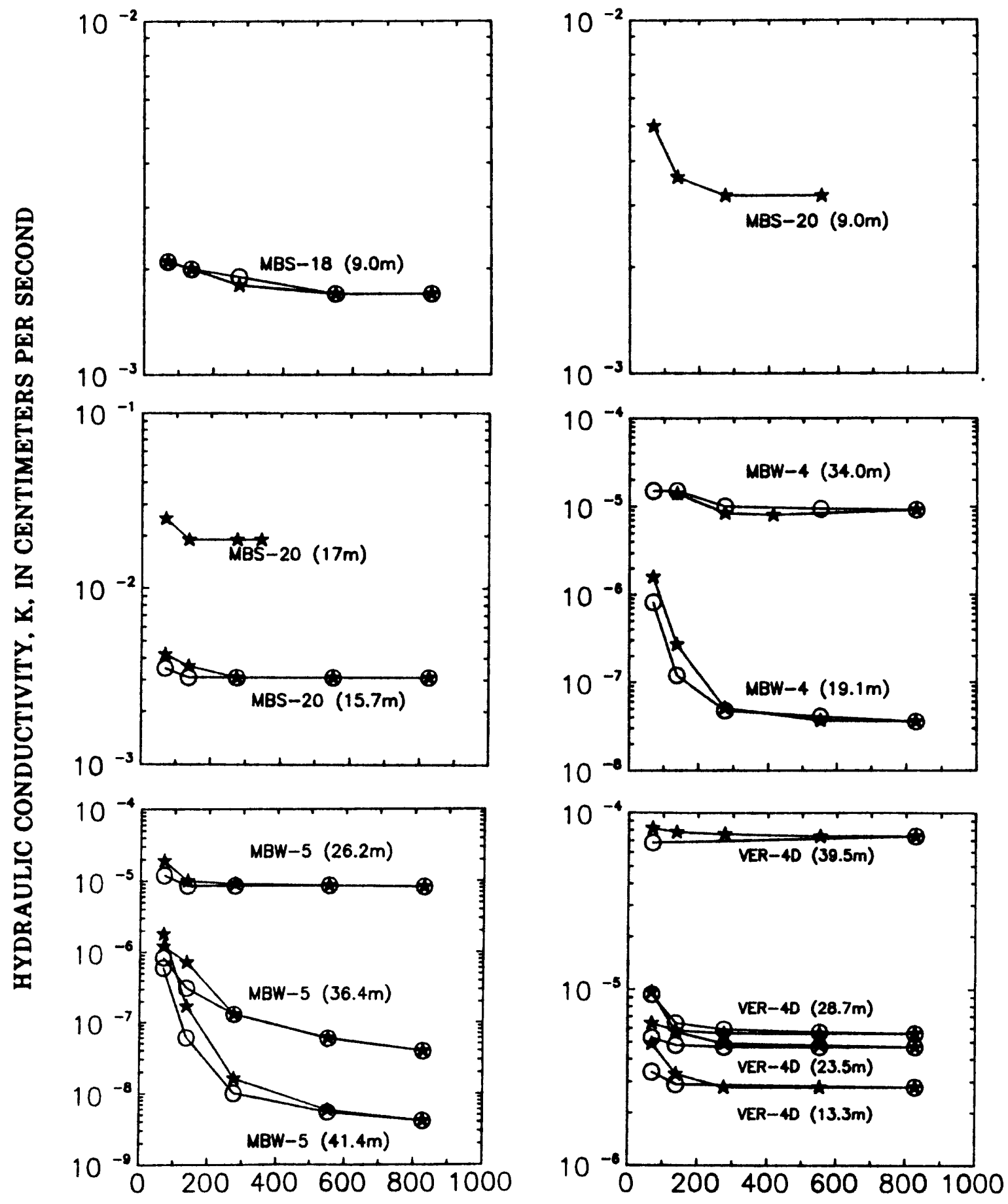

EFFECTIVE STRESS, IN KILOPASCALS

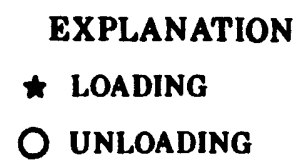

MBW-5 (41.4m) DRILL HOLE IDENTIFIER--

Depth, in meters 

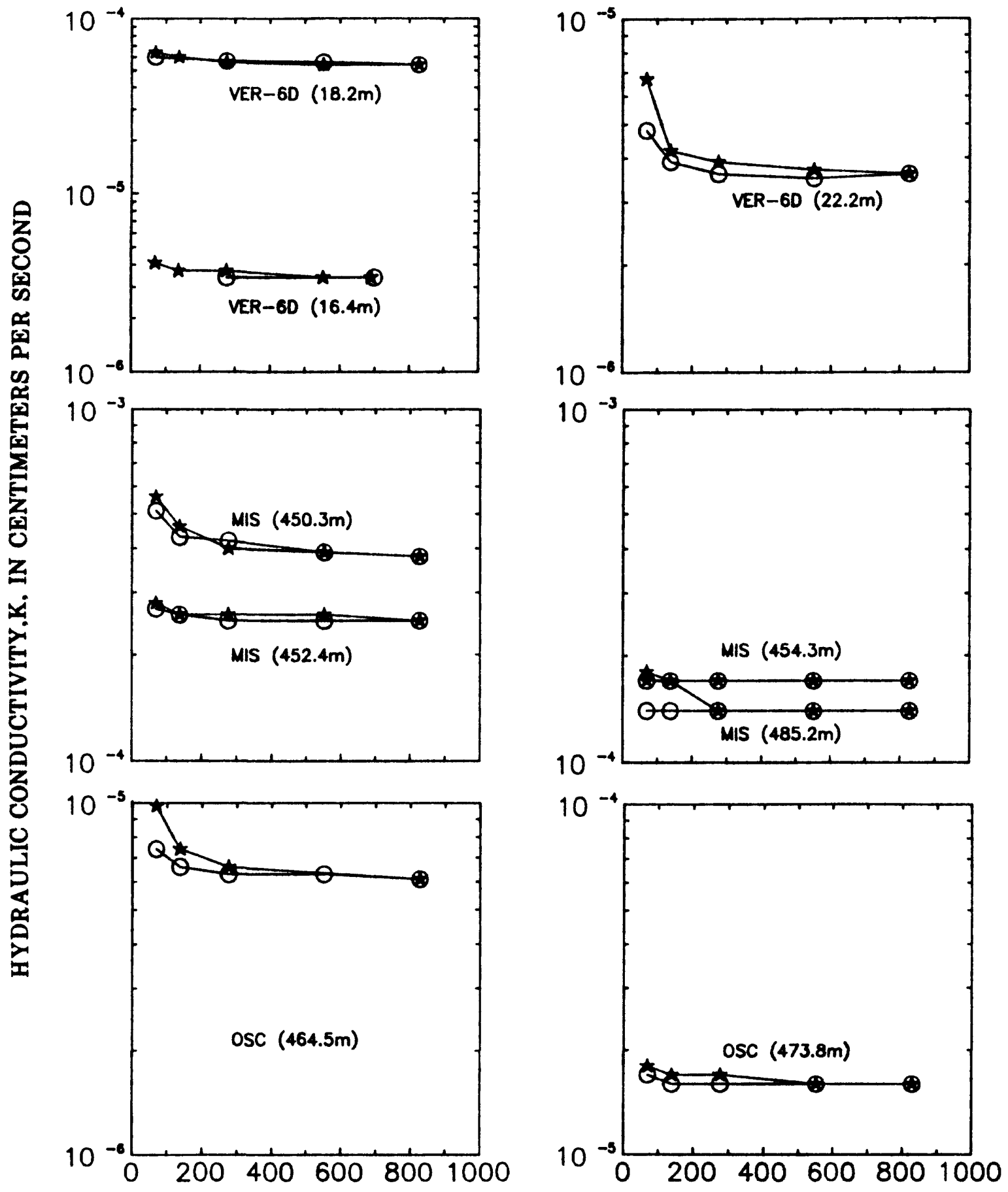

EFFECTIVE STRESS, IN KILOPASCALS

\author{
EXPLANATION \\ * LOADING \\ O UNLOADING
}

OSC $(473.8 \mathrm{~m})$ DRILL HOLE IDENTIFIER --

Depth, in meters 